Review

\title{
The Hippo pathway: a renewed insight in the craniofacial diseases and hard tissue remodeling
}

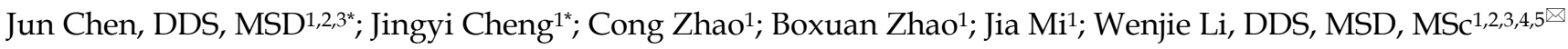 \\ 1. Xiangya School of Stomatology, Central South University, Changsha 410008, China. \\ 2. Xiangya Stomatological Hospital, Central South University, Changsha 410008, China. \\ 3. Hunan Key Laboratory of Oral Health Research, Hunan 3D Printing Engineering Research Center of Oral Care, Hunan Clinical Research Center of Oral \\ Major Diseases and Oral Health, Central South University, Changsha 410008, China. \\ 4. National Key Laboratory of Science and Technology on High-strength Structural Materials, Central South University, Changsha 410083, China. \\ 5. State Key Laboratory of Powder Metallurgy, Central South University, Changsha 410083, China. \\ *These authors contributed equally to this work.
}

$\triangle$ Corresponding author: Wenjie Li, DDS, MSD, MSc, Department of Orthodontics, Xiangya Stomatological Hospital, Xiangya School of Stomatology, Hunan Key Laboratory of Oral Health Research, Hunan 3D Printing Engineering Research Center of Oral Care, Hunan Clinical Research Center of Oral Major Diseases and Oral Health, Central South University, No. 72 of Xiangya Road, Changsha, 410008, P. R. of China. Telephone: +86-181-8212-9707 (Office), Fax: 0731-84805086. E-mail: liwenj@csu.edu.cn.

(c) The author(s). This is an open access article distributed under the terms of the Creative Commons Attribution License (https://creativecommons.org/licenses/by/4.0/). See http://ivyspring.com/terms for full terms and conditions.

Received: 2021.05.29; Accepted: 2021.09.20; Published: 2021.09.24

\begin{abstract}
The Hippo pathway plays an important role in many pathophysiological processes, including cell proliferation and differentiation, cell death, cell migration and invasion. Because of its extensive functions, Hippo pathway is closely related to not only growth and development, but also many diseases, including inflammation and cancer. In this study, the role of Hippo pathway in craniofacial diseases and hard tissue remodeling was reviewed, in attempting to find new research directions.
\end{abstract}

Key words: Hippo signaling pathway; YAP; TAZ; periodontal diseases; oral submucous fibrosis; craniofacial diseases

\section{Introduction}

Recently, the function of Hippo signaling pathway has attracted much attention in the craniofacial development and pathological processes. It has been proven that Hippo pathway is involved in the craniofacial inflammatory, cancerous and developmental diseases, such as periodontitis, odontogenic tumors, oral squamous cell carcinoma (OSCC) and tumor drug resistance. Hippo pathway also regulates the remodeling and development of craniofacial bone, the dysregulation of which will cause craniofacial deformity. This review will focus on summarizing recent advancements in two main sections: (1) Hippo pathway in the diseases of the craniofacial region, including oral submucous fibrosis (OSF), OSCC, odontogenic tumors, periodontitis, peri-implantitis and Sjogren's syndrome; (2) Hippo pathway in hard tissue remodeling, including periodontal tissue regeneration and orthodontic tooth movement (Figure 1). An overview of the specific upstream regulators and molecular downstream targets in these processes is also provided.

\section{An overview of Hippo pathway}

The Hippo pathway was first caught sight in Drosophila melanogaster by genetic mosaic screens for identifying growth suppressors [1, 2]. The key compositions of the pathway were highly conserved from Drosophila to mammals. Main components of canonical Hippo pathway in mammals include Mammalian Ste20-like protein kinase 1/2 (MST1/2, also known as STK4 and STK3), Salvador family WW domain-containing protein 1 (SAV1), Large tumor suppressor 1/2 (LATS1/2), MOB kinase activator 1A/B (MOB1a/b), and Yes-associated protein (YAP)/ Transcriptional co-activator with PDZ binding motif (TAZ) [3-5]. The core of Hippo pathway is the kinase cascade, which could be initiated by not only TAO kinases (TAOK1/2/3) that phosphorylated the 
activation loop of MST1/2, but also the autophosphorylation of MST1/2 through dimerization [6, 7]. The activated MST1/2 then phosphorylated SAV1 and MOB1a/b to phosphorylate and activate LATS1/2 [8-10]. Additionally, recent studies found that mitogenactivated protein kinase kinase kinase kinase (MAP4K) and Merlin (also named NF2) were also activators of LATS1/2 [11, 12]. MAP4Ks directly activated LATS1/2, while NF2 promoted the phosphorylation of LATS1/2 through MST1/2-SAV complex. The striatin-interacting phosphatase and kinase (STRIPAK) was recently reported to integrate upstream signals to activate MST1/2 and the MAP4Ks [13, 14]. Subsequently, activated LATS1/2 could phosphorylate transcriptional co-activators YAP and TAZ, the phosphorylation of which led to sequestration of YAP/TAZ in the cytoplasm by binding with 14-3-3 protein [15]. Phosphorylated YAP/TAZ detained in the cytoplasm finally were degraded by recruiting E3 ubiquitin ligases or autophagy [16, 17]. Hence, the purpose of the Hippo kinase cascade's terminal physiological output is to restrict the transcriptional activity of $\mathrm{YAP} / \mathrm{TAZ}$ by preventing YAP/TAZ from entering the nucleus. When the pathway was inactive, unphosphorylated YAP/TAZ accumulated in the nucleus and bounded the transcriptional enhancer associate domain transcription factors (TEADs, including TEAD1-4. Each TEAD had tissue-specific functions during development, such as cardiogenesis [18], neural development [19], myogenesis [20] and trophectoderm lineage determination [21]) to promote the expression of target genes such as CTGF, CYR61, ANKRD1, Col8a1 and etc. [22], facilitating cell proliferation, survival, and migration (Figure 2). In recent years, with the deepening of research, Hippo pathway has expanded into a complex signal network with more than 30 components. YAP and TAZ are considered as the canonical effectors of the pathway, responding to various intrinsic and extrinsic signals.

The canonical Hippo pathway regulates numerous biological processes, mainly including cell proliferation and apoptosis. Knockout of MST1, MST2, SAV1 or YAP could lead to liver overgrowth and hepatocellular carcinoma by regulating hepatic cell proliferation [23, 24]. MST1 has been considered as a pro-apoptotic kinase, which stimulated apoptosis by phosphorylating Beclin1 and consequently promoting sequestration of $\mathrm{Bcl}-2$ and $\mathrm{Bcl}-\mathrm{xL}$ by Beclin1 to activate Bax [25]. YAP could enhance P53 deacetylation, thus promoting cell survival by repressing P53-induced G0/G1 arrest and apoptosis [26]. YAP/TEADs were also associated with the epithelial-to-mesenchymal transition (EMT) transcription factor SLUG to directly inhibit proapoptotic BMF, repressing drug-induced apoptosis [27]. Some direct target genes of YAP/TAZ, such as Diap1 and BIRC3, have been identified as inhibitors of apoptosis [28]. In terms of mechanism, YAP could interact with and stabilize p73 to induce the apoptosis in DNA damage [29]. We summarized biological processes and diseases regulated by Hippo pathway, as shown in Table 1.

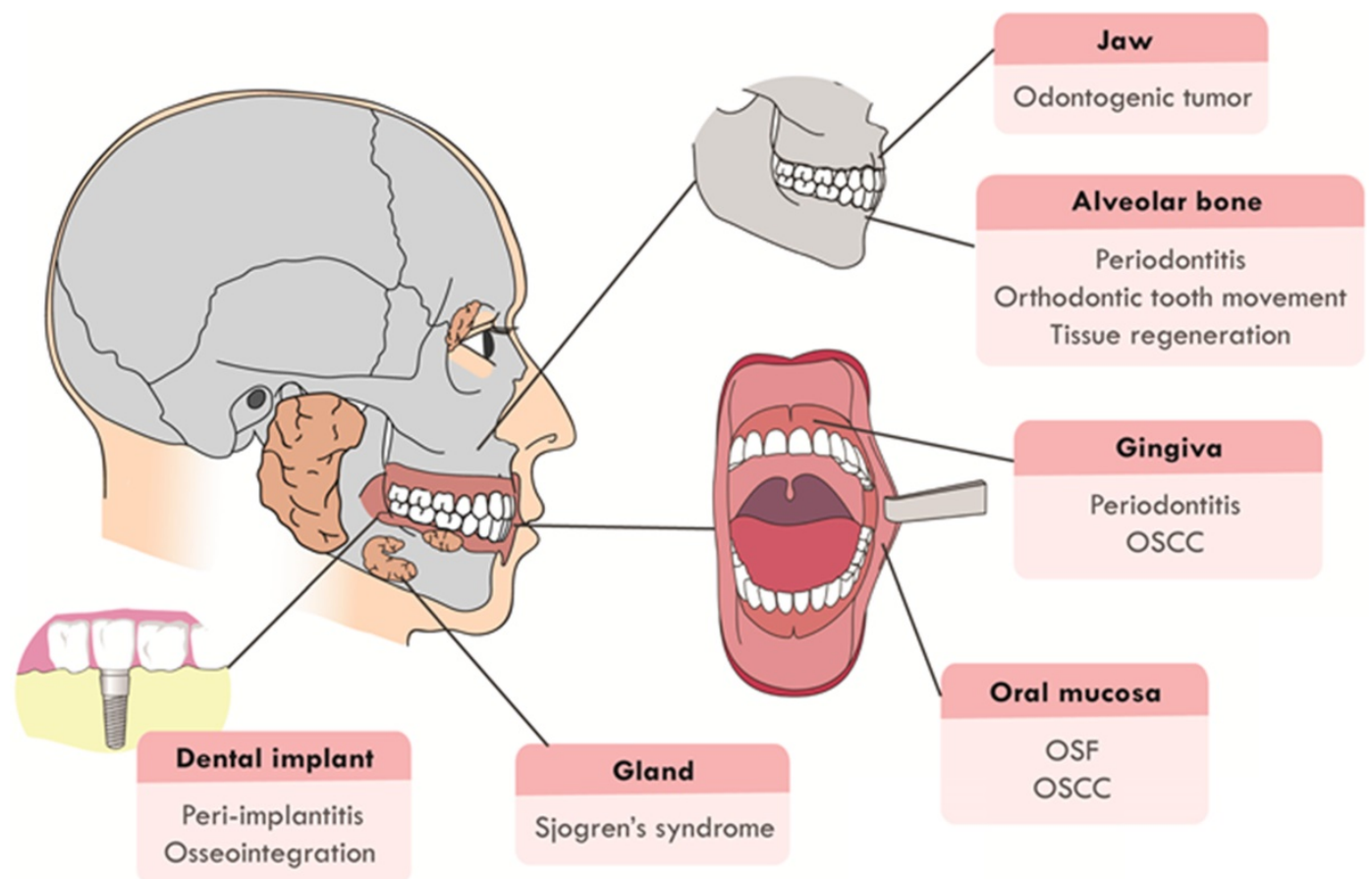

Figure 1. The craniofacial diseases and hard tissue remodeling that Hippo pathway is involved in. Hippo pathway plays a role in craniofacial diseases, including OSF, OSCC, odontogenic tumors, periodontitis, peri-implantitis, Sjogren's syndrome and periodontal hard tissue remodeling. 


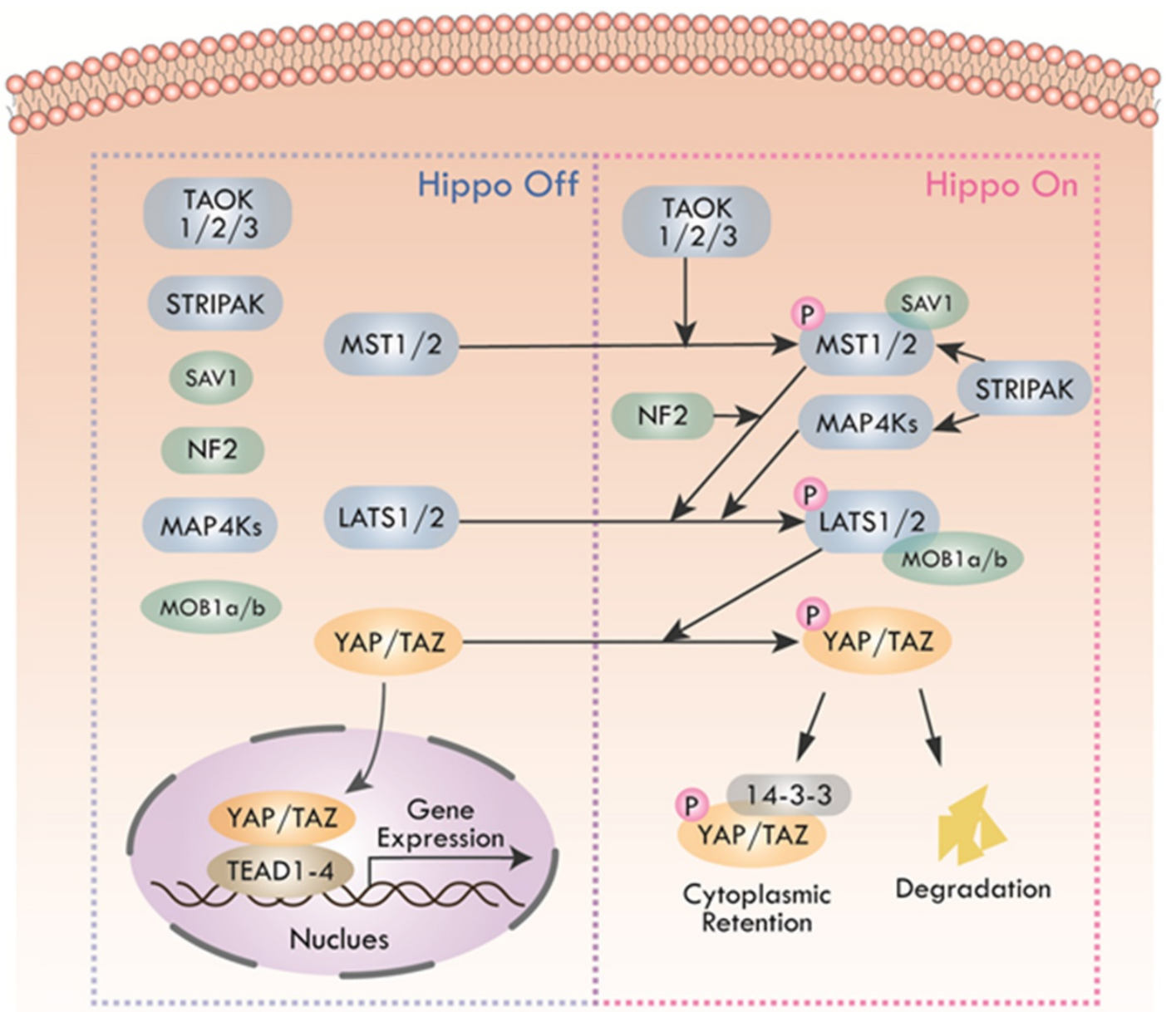

Figure 2. Canonical Hippo pathway in mammals. When Hippo pathway is inactivated, YAP and TAZ are dephosphorylated and activated, entering the nucleus and binding with TEADI-4 to activate downstream gene transcription. Hippo pathway, triggered by TAO kinase, phosphorylates MST1/2 with the help of scaffold proteins SAV1, MOB1a/b and NF2, thus affecting the phosphorylation of LATS1/2. MAP4Ks play similar roles with MST1/2. STRIPAK integrates upstream signals to activate MST1/2 and MAP4Ks. Activated LATS1/2 phosphorylate YAP/TAZ, leading to YAP/TAZ cytoplasmic retention by 14-3-3 protein or degradation.

Table 1. Biological processes and diseases regulated by Hippo pathway

\begin{tabular}{|c|c|c|}
\hline $\begin{array}{l}\text { Biological processes regulated by Hippo } \\
\text { pathway }\end{array}$ & Related diseases & Reference \\
\hline Cell proliferation & Liver overgrowth, Hepatocellular carcinoma, Colorectal cancer, Hypertrophic cardio-myopathy & {$[23,24,50,127-129]$} \\
\hline Cell cycle & OSCC, Pancreatic cancer & {$[55,130]$} \\
\hline Cell apoptosis & Breast cancer, Non-small-cell lung cancer, Hematological malignancies & {$[51,52,131]$} \\
\hline Cell autophagy & Aggressive breast cancers, Hepatocellular carcinoma, Thyroid cancer & {$[132-134]$} \\
\hline Cell senescence & Breast cancer, Osteoarthritis & {$[135,136]$} \\
\hline EMT & Organ fibrosis and cancer (lung, kidney, skin, liver) & [48] \\
\hline Angiogenesis & Cholangiocarcinoma, Pancreatic ductal adenocarcinoma & {$[137,138]$} \\
\hline Drug resistance of tumor cell & Breast cancer, Colorectal cancer, Esophageal cancer & {$[63,139,140]$} \\
\hline
\end{tabular}

In addition to the canonical pathway of regulating cell proliferation and apoptosis by inhibiting the entry of downstream transcriptional co-activator YAP into the nucleus, more and more studies have shown that Hippo pathway, with MST1/2 kinase as the core, has a variety of non-canonical regulatory functions. MST1 could mediate oxidative-stress-induced cell death by directly phosphorylating FOXO1/3 to promote FOXO1/3 nuclear translocation in neurons $[30,31]$. MST1/2 could also enhance FOXO1/3 stability through phosphorylation to promote the development of regulatory $\mathrm{T}$ cells and thus inhibit autoimmunity [32]. MST1 could directly phosphorylate IRF3, a key transcriptional factor of the antiviral-sensing pathway to inhibit the activation of IRF3 homodimerization and IRF3-mediated transcriptional responses. MST1 could also attenuate IRF3 activation by hindering virus-induced activation of TANK-binding kinase 1 (TBK1), thereby impeding cellular antiviral responses [33]. The studies suggest a negative regulation of MST1 in autoimmunity and host defense through non-canonical pathway.

Mechanical signals, stress signals and cell 
polarity are upstream signals to regulate Hippo pathway [34, 35]. On the one hand, YAP/TAZ were proven to play a role in cell type-specific differentiation of mesenchymal stem cells (MSCs) induced by extracellular matrix (ECM) stiffness. ECM stiffness mimicking the natural bone environment could promote the expression of YAP to induce the osteogenic potential of MSCs and enhance the ability of osteogenic differentiation, while soft ECM induced MSCs to differentiate into other cell types, such as adipocytes. Rho-GTPases and the actin cytoskeleton were essential for the nuclear localization of YAP/ TAZ during the mechanotransduction linked with ECM stiffness [36]. High cell density could also lead to the growth inhibition of cells through Hippo pathway [15]. Activation of Hippo kinase cascade in high cell density was associated to the trans-dimerization of E-cadherin at adherens junctions (AJs) [37], as well as the angiomotin (AMOT) complex in the tight junctions (TJs) [38]. On the other hand, Hippo pathway could respond to internal and environmental stressors that interfere with the normal state. When cells were faced with energy deficiency, the cellular energy stress led to increased AMP/ATP ratio, which activated the energy sensor AMP-activated protein kinase (AMPK). Activation of AMPK activated LATS1/2 or directly phosphorylated YAP, thus disrupting YAP-TEADs interaction and restoring energy homeostasis $[39,40]$. Other stress signals, such as oxidative stress [41] and cytokinesis failure [42] were also proved to activate Hippo signaling. It has been proved that cell polarity and adherence signals could activate the Hippo pathway. Many cell polarity components (Mer-Ex-Kibra complex, the apical transmembrane protein Crumbs, the Fat-Dachsous complex, etc.) have been demonstrated to regulate YAP/TAZ activity through Hippo pathway [34]. The Hippo signaling could also be initiated by components of junction complexes, including a-catenin, tyrosine-protein phosphatase non-receptor type 14 (PTPN14) and E-cadherin [43].

\section{Hippo pathway in the craniofacial diseases}

\section{Hippo pathway in oral precancerous lesions}

OSF is an oral precancerous lesion characterized by abnormal collagen deposition, closely related to betel nut chewing and commonly found in the Hunan Province of mainland China, India and Southeast Asia [44]. In arecoline treated endothelial cells, YAP was up-regulated with an enhanced transcriptional activity, which induced the activation of EMT. Abnormal deposition of Type I and III collagen is an important pathological feature of OSF, and knocking down of YAP can inhibit the secretion of Type I and II collagen. YAP-induced EMT was considered as a crucial event in OSF [45]. It has been proven that knockdown of bone morphogenetic protein 4 (BMP4) could affect the expression of EMT markers and inhibit ECM accumulation. Studies have shown that BMP4 as the downstream mediator was induced by the activation of YAP, which induced EMT in OSF [46]. The role of YAP on EMT in OSF suggested the potential target therapeutic value of YAP. The mechanical stimulation of oral mucosa caused by betel nut chewing also could lead to YAP activation, which promoted keratinocyte proliferation in OSF [47]. The mechanical activation of YAP/TAZ caused by increased matrix stiffness in tissue fibrosis could promote proliferation and survival of epithelial cells, which is crucial to the malignant transformation of OSF [47, 48]. However, the function of other components of Hippo pathway in the development of OSF, and the exact mechanism of Hippo pathway in the pathogenesis of OSF still need further elucidation. Until now, there are few studies on the role of Hippo pathway in other oral precancerous lesions such as oral leukoplakia, erythema and lichen planus. The relevant mechanisms remain to be supplemented, which may provide new clues for targeted therapy and recurrence prevention of oral premalignancy diseases.

\section{Hippo pathway in OSCC}

Hippo signaling pathway is known for regulating the occurrence and development of cancer. Knockout of MST1, MST2, SAV1 or YAP could lead to liver overgrowth and hepatocellular carcinoma [23, 24]. MST1, MST2 or SAV deletion could activate YAP to increase the intestinal crypt proliferation and tumorigenicity [49]. Studies of human colorectal cancer also have shown that the YAP/TAZ were closely related to tumor growth [50]. In addition, YAP/TAZ could inhibit apoptosis in the development of various cancers, such as breast cancer and non-small-cell lung cancer [51, 52]. A few direct target genes of YAP/TAZ, such as Diap1 and BIRC3, have been identified as inhibitors of apoptosis [28]. Collectively, Hippo pathway is closely related to carcinogenesis due to uncontrolled cell proliferation and apoptosis. Additionally, stromal stiffness in solid tumor tissues activated Hippo pathway as a type of mechanical signals. YAP has been proven to be activated in cancer-associated fibroblasts (CAFs) in response to mechanical stress and perturbation of the actin cytoskeleton, which established a positivefeedback loop to maintain the CAF phenotype [53].

Recently, the role of Hippo pathway in OSCC has also attracted attention. TP53, FAT1, PTEN, and 
EGFR were identified as upstream regulators of YAP, whereas TP63 was considered as a downstream effector of YAP. OSCC patients usually carry mutations in TP53, FAT1, PI3K/PTEN or EGFR signaling. These mutations could improve the activity of YAP in OSCC [54]. The YAP hyper-activation has been found to play a role in the early onset and rapid progression of OSCC, promoting tumorigenic phenotypes in OSCC cells and being essential to the development and metastasis of OSCC [55]. MiR-130a was highly expressed in OSCC cell lines. Overexpression of miR-130a promoted OSCC cell proliferation, metastasis and invasion by inactivating the Hippo signaling and activating YAP [56]. YAP could also be triggered by the over-expression of phosphatidylinositol 3-kinase catalytic subunit alpha (PIK3CA) [57], one of the most common oncogenic events in multiple malignancies including OSCC [58]. One study reported that Piezo-type mechanosensitive ion channel component 1 (PIEZO1), a Ca2+ channel, was a transcriptional target of YAP, regulating OSCC cellular growth [59]. TAZ has been revealed to promote the proliferation, migration, invasion, EMT and cancer stem cell maintenance of OSCC cells [60]. Studies have shown that TAZ enhanced the selfrenewal and maintenance of cancer stem cells (CSCs) by directly transcriptional activating downstream sex determining region $\mathrm{Y}$ box 2 (SOX2), the key transcriptional factor regulating CSCs properties from diverse cancer origins including OSCC [61]. Chronic mechanical damage of oral mucosa caused by long-term denture stimulation was a risk factor of OSCC. A recent study suggested that Hippo pathway acted as a mediator between denture-induced mechanotransduction and carcinogenesis. In the masticatory movements of denture wearer patients, mechanical stresses (shear, compressive, and tensile) transferred directly to palatal and alveolar mucosa, which was regulated by Hippo pathway and caused subsequent YAP/TAZ activation, promoting the symbolic biological behavior of cancer such as proliferation, survival, invasion, migration and angiogenesis [62]. In a word, the cascade reaction of Hippo pathway could exert a vital function in the onset and progression of OSCC.

Hippo pathway also plays a role in drug resistance of some cancers. Studies have shown that when YAP/TAZ were activated, tumor cells acquire the ability of chemotherapeutic drug resistance. TAZ could sustain the survival of breast cancer stem cells when treated with conventional chemotherapeutics such as paclitaxel and doxorubicin [63]. In addition, YAP/TAZ activation protected multiple tumor types from DNA damaging agents, including cisplatin, UV, and radiation [64-66]. YAP could also make tumor cell lines with BRAF, KRAS or NRAS mutations resistant to RAF and MEK inhibitor therapy [67]. Recently, the role of YAP on the drug resistance of OSCC has also been explored. Activation and nuclear translocation of YAP contributed to the acquisition of cisplatin resistance in OSCC [68]. One study showed that Ribosomal binding protein 1 (RRBP1) induced cisplatin resistance in OSCC by up-regulating YAP expression [69]. YAP over-expression led to gefitinib resistance in OSCC cells [70]. YAP has been suggested as a biomarker of treatment response in OSCC [70, 71].

Furthermore, studies have shown YAP/TAZ activation was associated with poor prognosis for OSCC. YAP activity was correlated with malignant phenotypes and poor prognosis [55, 72]. Pyruvate kinase M2 (PKM2), the key rate-limiting enzyme of glycolysis, highly expressed in oral tongue squamous cell carcinoma (OTSCC) tissues, maintaining the Warburg effect in tumor cells. The higher PKM2 expression was demonstrated to be related with a higher Tumor-Node-Metastasis (TNM) stage and a shorter overall survival. PKM2 knockdown could inhibited the proliferation and increased the apoptosis of OTSCC cells by activating Hippo signaling pathway, as confirmed by the decreased expression of YAP [73]. TAZ over-expression was also relevant to higher pathological grade, lymph node metastasis and poor prognosis $[60,74]$.

Due to the important role of YAP/TAZ in the pathogenesis, drug resistance and prognosis of OSCC, target for the components of Hippo pathway may probably bring new therapies for OSCC.

\section{Hippo pathway in odontogenic tumors}

Odontogenic tumor is a kind of tumor derived from odontogenic tissue, which includes enamel organ, dental sac and dental papilla. Keratocystic odontogenic tumor (KCOT) has been reported to be the most common odontogenic tumors in China [75], with some particular histologic features like parakeratinized stratified cell layers and daughter cysts [76]. In KCOT, YAP/TAZ were involved in the pathogenesis and proliferative growth. Compared with normal tissues, YAP/TAZ and downstream proteins (CYR61 and CTGF) were significantly up-regulated in KCOT [77]. In KCOT, the crosstalk between YAP/TAZ and Ki-67 has also been reported, revealing the Hippo signaling-mediated proliferative behavior [77]. In addition, YAP was over-expressed in odontogenic epithelium of ameloblastoma compared with the epithelial islands of dental follicle. YAP was considered to be essential for the neoplastic nature of ameloblastoma, and contribute to tumor invasiveness [78]. Although emerging studies have suggested the 
Hippo pathway was involved in odontogenic tumors, the exact mechanism still needs further investigations.

\section{Hippo pathway in periodontitis}

In recent years, more and more researches have confirmed that Hippo pathway was involved in the regulation of inflammation. Hippo pathway plays a pro-inflammatory role by inhibiting the activity of YAP, which exerts an anti-inflammatory effect.

Pathogen-associated molecular patterns (PAMPs) are key molecules that trigger inflammation in inflammatory processes, including periodontitis $[79,80]$. PAMPs could be recognized by multiple pattern recognition receptors (PRRs) like toll-like receptors (TLRs) to activate downstream inflammatory cascades [81, 82]. After TLRs recognizing PAMPs, the cytoplasmic Toll/interleukin (IL) 1 receptor (TIR) domains of the TLRs recruit the signaling adaptors, including MyD88, TIRAP, TRAM, and/or TRIF. According to the selection of specific adapter, various kinases (IRAK4, IRAK1, IRAK2, TAK1, TBK1, IKKe, etc.) and ubiquitin ligases (TRAF6, pellino 1, etc.) are activated to activate NF- $\mathrm{kB}$, type I interferon, MAPK and JNK pathways, leading to the expression and release of pro-inflammatory cytokines $[81,82]$. Inflammatory cytokines, including TNF-a and IL-1 $\beta$ could not only initiate Hippo pathwayinduced YAP/TAZ degradation but also TAK1mediated YAP/TAZ degradation. Furthermore, YAP impeded TAK1 substrate accessibility to prevent the downstream IKKa/ $\beta$ activation, thus inhibiting NF-kB signaling [83].

Lipopolysaccharides (LPS) is a typical kind of PAMPs, which is the cause of many inflammatory diseases, including periodontitis. In the LPS-triggered cascade of mammalian endothelial cells, YAP could directly bind to TRAF6, promote K48 ubiquitination to degrade TRAF6, and inhibit K63 ubiquitination to block the activation of downstream protein TAK1, thereby inhibiting the NF-KB signaling pathway and alleviating inflammatory response [84]. Additionally, mice with a lack of YAP/TAZ in the alveolar epithelial type II cells (AEC-IIs) showed extended inflammatory responses during bacterial pneumonia, revealing the anti-inflammatory function of YAP/ TAZ. Nuclear YAP/TAZ expression was significantly increased in AEC-IIs infected by Gram-positive pathogen Streptococcus, and then YAP/TAZ-induced IKBa expression was enhanced, which suppressed NF-KB response [85] (Figure 3).

Alveolar bone loss is a hallmark of periodontitis progression [86]. Hippo pathway has been reported as a biochemical signal response effector in bone remodeling regulation. Hippo pathway could transform inflammatory stimuli induced by periodontitis into regulation of osteoblasts and osteoclasts activity. NF- $\mathrm{KB}$ receptor activator (RANK) and NF-KB ligand receptor activator (RANKL) jointly promote differentiation, maturation and activation of osteoclasts and progenitor cells during inflammatory bone remodeling, while osteoprotegerin (OPG) blocks this process and inhibits osteoclasts differentiation, thereby alleviating bone damage $[87,88]$. In response to inflammatory stimuli, YAP not only decreased the expression of RANKL, but also enhanced the expression of OPG in osteoblast to resist inflammatory bone resorption [89]. Additionally, a-calcitonin gene-related peptide (aCGRP) could up-regulate the expression of osteogenic phenotype in human periodontal ligament cells (hPDLCs) by activating YAP [90]. However, one study reported a contrary finding that periodontitis with traumatic occlusion (TO) is a condition in which inflammatory stimuli and mechanical stress signals work together, and that YAP can increase bone resorption, but the exact mechanism remains unclear [91].

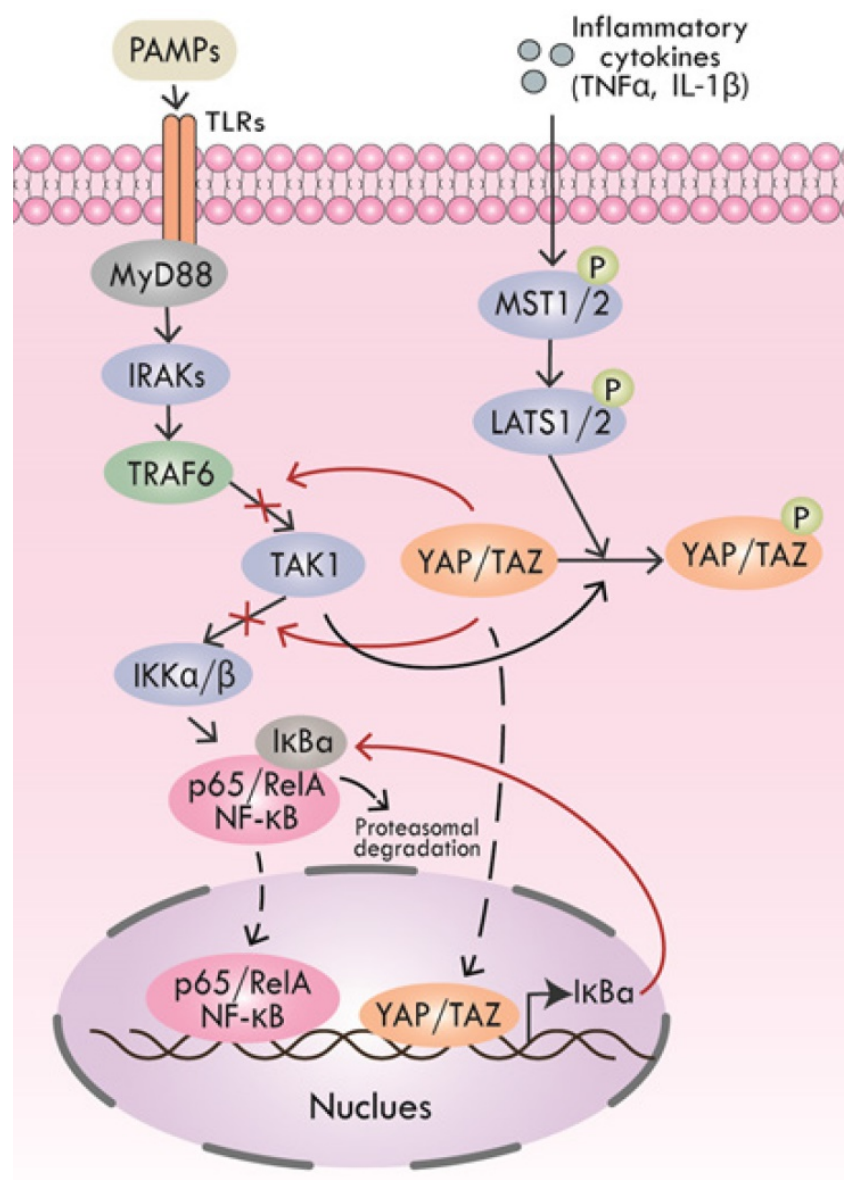

Figure 3. Regulation of Hippo pathway on inflammatory signals. YAP and TRAF6 synergistically inhibit the activation of downstream protein TAK1, thereby suppressing NF-KB signaling. YAP also interacts with TAK1 to inhibit downstream IKK $\alpha / \beta$ activation, thus inhibiting NF-KB signaling. TNF- $\alpha$ and IL- $1 \beta$ initiate YAP/TAZ degradation through Hippo signaling. Inhibitory IKBa expression induced by nuclear YAP/TAZ inhibits NF-KB response. 
Angiogenesis and bone immune are two important events in periodontitis [92, 93]. Angiogenesis is the key biological processes in periodontal tissue and organ reconstruction [94]. YAP/TAZ have been proved as a key regulator of angiogenesis and vascular barrier maturation by activating actin cytoskeleton remodeling and endothelial cell (EC) proliferation [95]. VEGF could activate YAP/TAZ by acting on the actin cytoskeleton, triggering transcriptional programs to control cytoskeleton dynamics, thereby establishing feedforward cycles to ensure appropriate angiogenic response [96]. VEGF/VEGFR could also inhibit MST1/2 via PI3K/MAPK to impede Hippo signaling and activate YAP/TAZ [97]. These results have indicated that YAP/TAZ acted as downstream effectors of VEGFR. Furthermore, YAP/TAZ were demonstrated to initiate angiogenesis through YAP/ TAZ-ANG-2/CYR61 axis [97].

The concept of osteoimmunology was first proposed by Arron et al., declaring the correlation between bone and immune system. Bone cells participate in immune regulation by secreting cytokines. Abnormal activity of immune cells results in changes in osteogenesis and angiogenesis[98]. Macrophage is the major participant in bone immune response. Studies have shown that Hippo-YAP pathway plays a role in the polarization of macrophage to M1/M2 phenotypes. Wnt5a promoted TGF 31 -mediated M2 polarization by inducing YAP/TAZ [99]. The inhibition of Hippo pathway in macrophage enhanced not only YAP nuclear translocation but also interaction with $\beta$-catenin, which could impede XBP1-mediated NLR family pyrin domain containing 3 (NLRP3) activation, resulting in IL-1 $\beta$ release reduced and M2 macrophage phenotype promoted [100]. However, some studies have shown opposite results. For example, YAP was reported to impair M2 but facilitate M1 macrophage polarization. Myeloid YAP knockout resulted in increased M2 phenotypic IL-10 and decreased M1 phenotypic IL-1 $\beta$ [101]. aCGRPYAP signaling was proved to inhibit osteogenic factors secretion of M2 macrophages at first, and then promote the osteogenic factor secretion by regulating the bone immune response of M2 macrophages [102].

As mentioned above, although studies on the role of Hippo-YAP pathway in angiogenesis and bone immunity have been in-depth, there are still few studies on the role of Hippo pathway in regulating periodontitis through angiogenesis and bone immunity. Therefore, studies in this field can be considered in the future. In addition, whether Hippo pathway regulates periodontal ligament regeneration needs further study.

\section{Hippo pathway in peri-implantitis}

Hippo-YAP pathway has also been revealed to be involved in the regulation of peri-implantitis. Titanium (Ti) and its corresponding alloys have been widely used in dental implants. Ti ions can be released from the implant into surrounding tissues in the presence of host detrimental electrolytic aqueous environment [103]. In the early stage of Ti exposure, NF-kB pathway was activated by up-regulating the nuclear expression of $\mathrm{YAP}$, triggering an inflammatory response and resulting in tissue damage adjacent to oral implants. In this process, YAP over-expression enhanced Ti-induced inflammatory response through NF-kB pathway. However, the continuous activation of NF- $\mathrm{kB}$ pathway inhibited the expression of YAP and thus suppressed the inflammatory response, which acting as a negative feedback[104]. In addition, Ti ions could also inhibit osteogenic differentiation of osteoblasts by increasing YAP nuclear expression [103]. Taken together, the promotion of inflammatory response and inhibition of osteogenesis in peri-implantitis induced by Ti ions were both associated with the YAP over-expression.

\section{Hippo pathway in Sjogren's syndrome}

Sjogren's syndrome (SS) is a complex autoimmune disease primarily affecting salivary and lacrimal glands. Although immune deficiency is considered to be the main cause of SS, increasing studies indicate that loss of cell polarity and structural integrity, including E-cadherin adhesion deficiency, play an important role in SS. E-cadherin has been shown to be an important regulator of morphologic transformation during submandibular gland (SMG) development, which could establish the apical-basal polarity in acinar and ductal precursor cells [105]. Recently, studies have shown that the E-cadherin junctions can interact with the Hippo pathway, which is necessary for SMG branching morphogenesis. These findings from the mouse model were validated in human labial biopsy specimens from patients with SS [106]. The interaction of TAZ with E-cadherin and a-catenin, two major determinants of cell polarity, was essential for normal gland cell differentiation and structure organization [106]. Moreover, YAP was involved in the formation of SMG epithelial progenitor cells by inducing Epiregulin expression, and dysregulated YAP-mediated cell fate control was thought to be related to SS [107]. 


\section{Hippo pathway in hard tissue remodeling}

\section{Hippo pathway in periodontal tissue regeneration}

Periodontal tissue contains alveolar bone, gingiva, periodontal ligament and cementum. The main function of the periodontal tissue is to support, fix and nourish teeth. Periodontal tissue regeneration is of great significance to the treatment of periodontal diseases. Recent studies have shown that Hippo pathway acts as an important regulator in periodontal tissue regeneration.

Human periodontal ligament stem cells (hPDLSCs) are considered as the most promising seed cells for periodontal tissue regeneration owing to their self-renewing and multi-lineage differentiation [108]. Activation of YAP could promote the proliferation of hPDLSCs, inhibit cell apoptosis, and delay cell senescence [109, 110]. Hippo pathway inactivation and YAP dephosphorylation could promote cell proliferation and inhibit apoptosis and senescence induced by telomerase reverse transcriptase (TERT) overexpression [111]. Over-expression of TAZ could promote the proliferation of hPDLSCs and inhibit apoptosis [112]. The crosstalk between Hippo and ERK or AKT has been considered as a vital process in periodontal tissue regeneration. When YAP was up-regulated, protein levels of P-Msk1, P-ERK1/2 and its target genes were increased [110], suggesting that YAP could activate ERK pathway. ERK and AKT pathway were involved in various biological processes, including the regulation of cell metabolism, proliferation and survival $[113,114]$. In hPDLSCs, the phosphorylation of ERK and AKT was affected by YAP, which was responsible for the regulation of the cell proliferation and cell senescence [111]. Studies have also found that YAP/TAZ can balance the process of osteogenic and adipogenic differentiation in hPDLSCs. The up-regulation of YAP promoted osteogenic differentiation and inhibit adipogenic differentiation, while knockdown of YAP led to an opposite result. In this process, YAP enhanced the stabilization and nuclear transfer of $\beta$-catenin, possibly by regulating upstream proteins of Wnt/ $\beta$-catenin pathway, including LRP6 and DVL3 [115]. Over-expression of TAZ also promoted osteogenic differentiation of hPDLSCs through p-SMAD3[112]. We summarized the signaling crosstalk of Hippo pathway in periodontal tissue regeneration, as shown in Table 2.

The regulatory role of YAP in other types of oral stem cells has also been reported. YAP mediated the mineralization induced by the static magnetic field in dental pulp stem cells (DPSCs) [116, 117]. MiR-141-3p inhibited proliferation and promoted senescence of stem cells from apical papilla (SCAPs) by post-transcriptionally downregulating YAP [118]. Interestingly, some studies have shown inconsistent results in the role of YAP in osteogenesis-related cells $[103,119]$. Some studies have shown that YAP can inhibit the osteoblastic activity of osteoblasts. The reason is that the WW domain of YAP interacts with the PY motif of Runx2 to form the YAP/ Runx2 complex, thus inhibiting the activity of Runx2 in osteoblasts $[119,120]$. Similar result of YAP inhibiting the osteogenic differentiation was also showed in mesenchymal stem cells (MSCs) [120]. However, physical stimulations could promote osteogenesis by activating YAP [121, 122].

Table 2. Signaling crosstalk of Hippo pathway in periodontal tissue regeneration

\begin{tabular}{lll}
\hline $\begin{array}{l}\text { Signaling crosstalk of the } \\
\text { Hippo signaling pathway }\end{array}$ & Related Cellular Processes & Reference \\
\hline Wnt $/ \beta$-catenin & Differentiation & \\
ERK & Proliferation, Apoptosis, Senescence & {$[115,141,142]$} \\
PI3K/AKT & Proliferation, Senescence & {$[110,112]$} \\
TGF- $\beta /$ SMAD & Proliferation, Differentiation & {$[112]$} \\
\hline
\end{tabular}

An in vivo study of oral implant bone healing showed that aCGRP could up-regulate the expression of YAP and aCGRP-YAP pathway could promote angiogenesis and osteogenesis during implant bone healing, especially in the early stage [123].

As crucial members of Hippo pathway, YAP and TAZ contributed to promoting proliferation, inhibiting apoptosis and senescence, as well as regulating osteogenic or lipogenic differentiation of many types of oral stem cells. Targeting YAP/TAZ to regulate the proliferation and differentiation of hPDLSCs through the Hippo pathway may provide a new strategy for periodontal tissue regeneration, which may be a direction of future research.

\section{Hippo pathway in orthodontic tooth movement}

Mechanical stress, as an important factor, could activate YAP/TAZ to induce osteogenic differentiation of hPDLCs, which contributes to the alveolar bone formation in orthodontic tooth movement (OTM). Cyclic stretch was found to induce YAP nuclear localization and activity, promoting the osteogenic differentiation of hPDLCs [124]. In a high-throughput sequencing study, the expression profile of stretched hPDLCs revealed several important components of the Hippo pathway such as LATS1, YAP and TEAD1/2 were up-regulated [125]. One study has shown that TAZ adjusted bone tissue remodeling through Runx2, while YAP regulated periodontal cell proliferation and differentiation during OTM [126]. In general, Hippo pathway acts as 
a mechanical-chemical signal transduction hub in OTM, which coordinates bone remodeling and tooth relocation. However, the study of Hippo pathway in OTM is still not in-depth, and future research can be strengthened in this aspect.

\section{Conclusion}

According to the existing literatures, Hippo pathway plays an important role in a slew of oral events, including periodontal tissue regeneration, periodontitis, peri-implantitis, SS, odontogenic tumors, OSF and OSCC. We summarized the research progress of Hippo pathway in oral diseases, as shown in Table 3 and Figure 4. To date, studies about Hippo pathway in periodontal tissue regeneration were mainly focused on the regulation of osteogenesis in
hPDLSCs, while the function of Hippo pathway in periodontal ligament regeneration still needs to be elucidated. The involvement of Hippo pathway in periodontitis by regulating angiogenesis and bone immunity is also worthy of further study. The role of Hippo pathway in peri-implantitis is still in its infancy. What's more, further researches on the role of Hippo pathway in the development of OSF are expected. Mechanism studies on the role of Hippo signaling in OSF and its carcinogenesis will help clarify whether Hippo pathway has the potential to be a new therapeutic target for OSF. Further researches can also focus on investigating the function of Hippo pathway in other oral precancerous lesions such as oral leukoplakia and erythema.

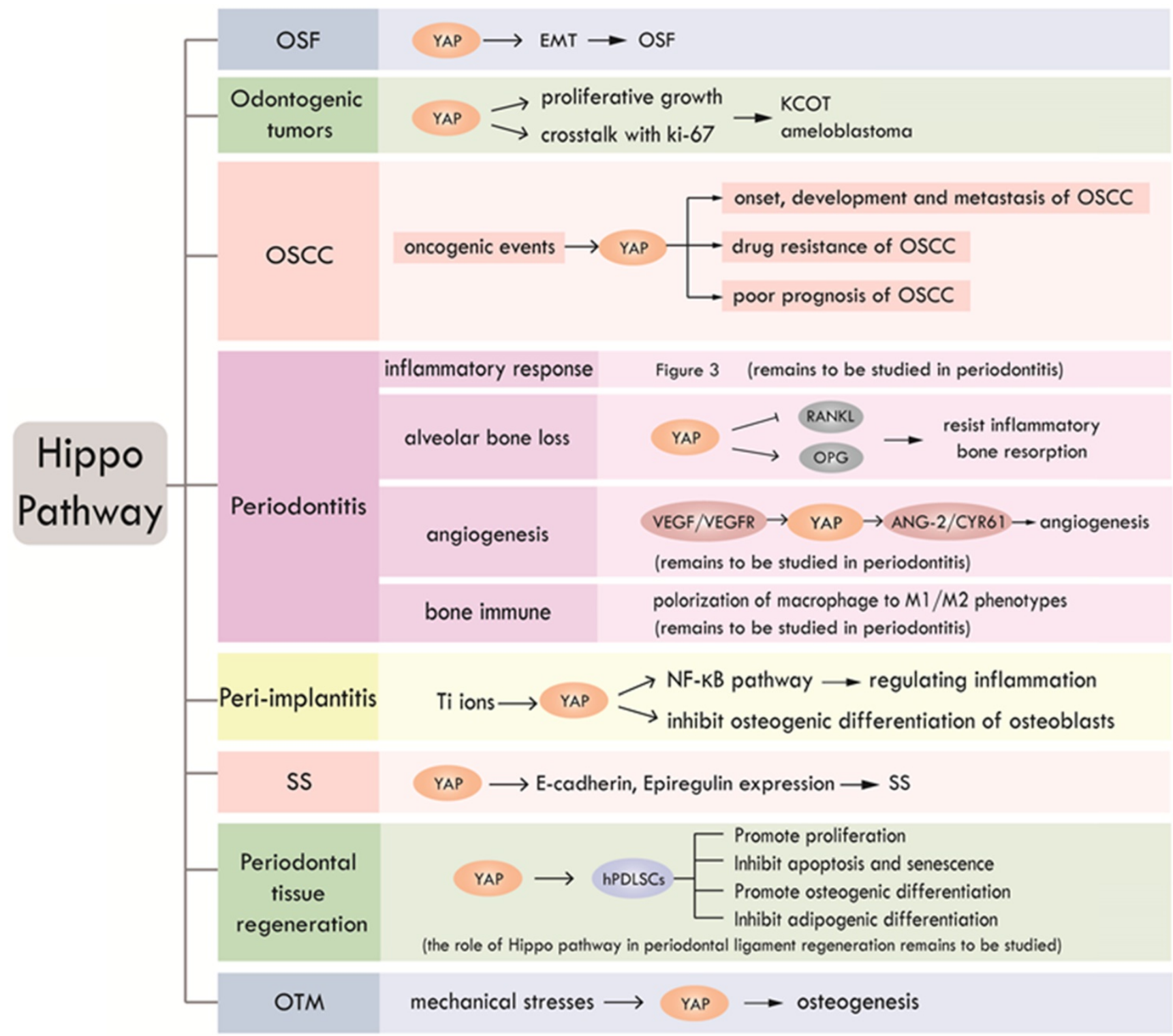

Figure 4. Research status and prospect of Hippo pathway in craniofacial diseases and hard tissue remodeling. Hippo pathway is involved in OSF, odontogenic tumors, OSCC, periodontitis, peri-implantitis, SS, periodontal tissue regeneration and OTM through different biological processes. 
Table 3. The role of Hippo pathway in oral diseases

\begin{tabular}{|c|c|c|c|c|c|}
\hline Oral Disease & Upstream Signals & $\begin{array}{l}\text { The effector in } \\
\text { Hippo pathway }\end{array}$ & $\begin{array}{l}\text { Activated/ } \\
\text { Inhibited }\end{array}$ & Effect & Reference \\
\hline \multirow[t]{2}{*}{ OSF } & arecoline & YAP & Activated & Promote EMT & {$[45]$} \\
\hline & mechanical stresses & & & Promote keratinocyte proliferation & [47] \\
\hline \multirow[t]{7}{*}{ OSCC } & $\begin{array}{l}\text { TP53/FAT1/PTEN/EG } \\
\text { FR/MiR-130a/PIK3CA }\end{array}$ & YAP & Activated & Promote proliferation, metastasis, invasion, etc. & {$[54,56,57]$} \\
\hline & mechanical stresses & & & & {$[62]$} \\
\hline & - & TAZ & & & [60] \\
\hline & RRBP1 & YAP & Activated & Promote cisplatin resistance & [69] \\
\hline & - & & & Promote gefitinib resistance & [70] \\
\hline & PKM2 & YAP & Activated & Promote the poor prognosis & [73] \\
\hline & - & TAZ & & & {$[60,74]$} \\
\hline Odontogenic tumor & - & YAP/TAZ & Activated & Promote proliferation, invasion, etc. & {$[77,78]$} \\
\hline Periodontitis & - & YAP & Activated & Resist inflammatory bone resorption & [89] \\
\hline Peri-implantitis & Ti ions & YAP & Activated & $\begin{array}{l}\text { Promote inflammatory responses; } \\
\text { Inhibit osteogenic differentiation of osteoblasts }\end{array}$ & {$[103,104]$} \\
\hline SS & - & YAP/TAZ & Activated & Promote gland formation & {$[105-107]$} \\
\hline Periodontal tissue regeneration & - & YAP & Activated & $\begin{array}{l}\text { In hPDLSCs: Promote proliferation; Inhibit apoptosis } \\
\text { and senescence; Promote osteogenic differentiation; } \\
\text { Inhibit adipogenic differentiation }\end{array}$ & $\begin{array}{l}{[109,110,} \\
115]\end{array}$ \\
\hline Implant osseointegration & aCGRP & YAP & Activated & Promote angiogenesis and osteogenesis & [123] \\
\hline Orthodontic tooth movement & mechanical stresses & YAP & Activated & Promote osteogenic differentiation of hPDLCs & [124] \\
\hline
\end{tabular}

To sum up, Hippo pathway is involved in various physiological and pathological processes of craniofacial diseases, a thorough study of which may help to better reveal the occurrence and development of craniofacial diseases and provide new ideas for the treatment.

\section{Abbreviations}

OSCC: oral squamous cell carcinoma; OSF: oral submucous fibrosis; MST1/2: mammalian Ste20-like protein kinase 1/2; SAV1: salvador family WW domain-containing protein 1; LATS1/2: large tumor suppressor 1/2; MOB1a/b: MOB kinase activator 1A/B; YAP: yes-associated protein; TAZ: transcriptional co-activator with PDZ binding motif; MAP4K: mitogen-activated protein kinase kinase kinase kinase; STRIPAK: striatin-interacting phosphatase and kinase; TEADs: transcriptional enhancer associate domain transcription factors; EMT: epithelial-to-mesenchymal transition; ER: endoplasmic reticulum; TBK1: TANK-binding kinase 1; MSCs: mesenchymal stem cells; ECM: extracellular matrix; AMOT: angiomotin; AMPK: AMP-activated protein kinase; PTPN14: tyrosine-protein phosphatase non-receptor type 14; BMP: bone morphogenetic protein; CAFs: cancer-associated fibroblasts; PIK3CA: phosphatidylinositol 3-kinase catalytic subunit alpha; CSCs: cancer stem cells; SOX2: sex determining region Y box 2; PAMPs: pathogen-associated molecular patterns; PRRs: pattern recognition receptors; TLRs: toll-like receptors; TIR: Toll/interleukin (IL) 1 receptor; IL: interleukin; AECIIs: alveolar epithelial type II cells; RANK: NF-KB receptor activator; RANKL: NF-kB ligand receptor activator; OPG: osteoprotegerin; aCGRP: a-calcitonin gene-related peptide; hPDLCs: human periodontal ligament cells; TO: traumatic occlusion; EC: endothelial cell; NLRP3: NLR family pyrin domain containing 3; SS: Sjogren's syndrome; SMG: submandibular gland; hPDLSCs: human periodontal ligament stem cells; TERT: telomerase reverse transcriptase; DPSCs: dental pulp stem cells; SCAPs: stem cells from apical papilla; MSCs: mesenchymal stem cells; OTM: orthodontic tooth movement.

\section{Acknowledgements}

We thank the enormous support from Xiangya School of Stomatology, Xiangya Stomatological Hospital, Central South University.

\section{Financial support}

This work was supported by Natural Science Foundation of Hunan Province $(\mathrm{CN})$ (Grant No: 2019JJ50784 to Wenjie Li), the Young Teacher's Institutional grant from Xiangya School of Stomatology and Xiangya Stomatological Hospital, Central South University (Grant No: 2018YQ02 to Wenjie Li), the Hunan Health Commission Research Grant, China (Grant No: 2020800 to Wenjie Li), Natural Science Foundation of Hunan Province (CN) (Grant No: 2020JJ5404 to Jun Chen), the Young Teacher's Institutional grant from Xiangya School of Stomatology and Xiangya Stomatological Hospital, Central South University (Grant No: 2019YQ01 to Jun Chen), the Hunan Health Commission Research Grant, China (Grant No: 202108011054 to Jun Chen), the College Students' Innovative Entrepreneurial Training Program (Grant No: 2021105330204 and S2020105330353). The funding provided economic support for the study in its research design and data 
collection. The funders had no role in study design, data collection and analysis, decision to publish, or preparation of the manuscript.

\section{Author Biography}

Dr. Weniie Li (DDS. MSD. MSc.) is the attending doctor at the Department of Orthodontics, Xiangya School of Stomatology, Central South University (Changsha, China). He received his post-doc training at the School of Dentistry (SoD), University of Washington (Seattle, WA, USA) from 2012-2016 and henceforth maintained a senior fellow position at the Department of Oral Health Science, SoD. His main research interests include biomaterials for dental and craniofacial tissue engineering, molecular developmental biology of craniofacial growth, genetic basis of craniofacial and dental abnormalities, evidence-based orthodontics, artificial intelligence assisted orthodontic diagnosis.

Dr. Jun Chen (DDS. MSD.) is a periodontal specialist at the Xiangya Stomatological Hospital, Central South University (Changsha, China). Dr. Chen also obtains the lecturer and attending doctor positions at the Xiangya School of Stomatology, Central South University (Changsha, China). Her main research interests are periodontal bone regeneration and targeted treatment of oral mucosal potential malignant diseases.

\section{Competing Interests}

The authors have declared that no competing interest exists.

\section{References}

1. Justice RW, Zilian O, Woods DF, Noll M, Bryant PJ. The Drosophila tumor suppressor gene warts encodes a homolog of human myotonic dystrophy kinase and is required for the control of cell shape and proliferation. Genes Dev. 1995; 9: 534-46.

2. Xu T, Wang W, Zhang S, Stewart RA, Yu W. Identifying tumor suppressors in genetic mosaics: the Drosophila lats gene encodes a putative protein kinase. Development. 1995; 121: 1053-63.

3. Halder G, Johnson RL. Hippo signaling: growth control and beyond. Development. 2011; 138: 9-22.

4. Pan D. The hippo signaling pathway in development and cancer. Dev Cell. 2010; 19: 491-505.

5. Yu FX, Zhao B, Guan KL. Hippo Pathway in Organ Size Control, Tissue Homeostasis, and Cancer. Cell. 2015; 163: 811-28.

6. Boggiano JC, Vanderzalm PJ, Fehon RG. Tao-1 phosphorylates Hippo/MST kinases to regulate the Hippo-Salvador-Warts tumor suppressor pathway. Dev Cell. 2011; 21: 888-95.

7. Glantschnig H, Rodan GA, Reszka AA. Mapping of MST1 kinase sites of phosphorylation. Activation and autophosphorylation. J Biol Chem. 2002; 277: 42987-96.

8. Callus BA, Verhagen AM, Vaux DL. Association of mammalian sterile twenty kinases, Mst1 and Mst2, with hSalvador via C-terminal coiled-coil domains, leads to its stabilization and phosphorylation. FEBS J. 2006; 273: 4264-76.

9. Praskova M, Xia F, Avruch J. MOBKL1A/MOBKL1B phosphorylation by MST1 and MST2 inhibits cell proliferation. Curr Biol. 2008; 18: 311-21.

10. Hergovich A, Schmitz D, Hemmings BA. The human tumour suppressor LATS1 is activated by human MOB1 at the membrane. Biochem Biophys Res Commun. 2006; 345: 50-8.

11. Meng Z, Moroishi T, Mottier-Pavie V, Plouffe SW, Hansen CG, Hong AW, et al. MAP4K family kinases act in parallel to MST1/2 to activate LATS1/2 in the Hippo pathway. Nat Commun. 2015; 6: 8357

12. Yin F, Yu J, Zheng Y, Chen Q, Zhang N, Pan D. Spatial organization of Hippo signaling at the plasma membrane mediated by the tumor suppressor Merlin/NF2. Cell. 2013; 154: 1342-55.
13. Chen R, Xie R, Meng Z, Ma S, Guan KL. STRIPAK integrates upstream signals to initiate the Hippo kinase cascade. Nat Cell Biol. 2019; 21: 1565-77.

14. Ardestani A, Maedler K. STRIPAK Is a Regulatory Hub Initiating Hippo Signaling. Trends Biochem Sci. 2020; 45: 280-3.

15. Zhao B, Wei X, Li W, Udan RS, Yang Q, Kim J, et al. Inactivation of YAP oncoprotein by the Hippo pathway is involved in cell contact inhibition and tissue growth control. Genes Dev. 2007; 21: 2747-61.

16. Liu CY, Zha ZY, Zhou X, Zhang H, Huang W, Zhao D, et al. The hippo tumor pathway promotes TAZ degradation by phosphorylating a phosphodegron and recruiting the SCF\{beta\}-TrCP E3 ligase. J Biol Chem. 2010; 285: 37159-69.

17. Liang N, Zhang C, Dill P, Panasyuk G, Pion D, Koka V, et al. Regulation of YAP by mTOR and autophagy reveals a therapeutic target of tuberous sclerosis complex. J Exp Med. 2014; 211: 2249-63.

18. Chen Z, Friedrich GA, Soriano P. Transcriptional enhancer factor 1 disruption by a retroviral gene trap leads to heart defects and embryonic lethality in mice. Genes Dev. 1994; 8: 2293-301.

19. Sawada A, Kiyonari H, Ukita K, Nishioka N, Imuta Y, Sasaki H. Redundant roles of Tead1 and Tead 2 in notochord development and the regulation of cell proliferation and survival. Mol Cell Biol. 2008; 28: 3177-89.

20. Figeac N, Mohamed AD, Sun C, Schonfelder M, Matallanas D, Garcia-Munoz A, et al. VGLL3 operates via TEAD1, TEAD3 and TEAD4 to influence myogenesis in skeletal muscle. J Cell Sci. 2019; 132.

21. Nishioka N, Inoue K, Adachi K, Kiyonari H, Ota M, Ralston A, et al. The Hippo signaling pathway components Lats and Yap pattern Tead4 activity to distinguish mouse trophectoderm from inner cell mass. Dev Cell. 2009; 16: 398-410

22. Zhao B, Ye X, Yu J, Li L, Li W, Li S, et al. TEAD mediates YAP-dependent gene induction and growth control. Genes Dev. 2008; 22: 1962-71.

23. Lu L, Li Y, Kim SM, Bossuyt W, Liu P, Qiu Q, et al. Hippo signaling is a potent in vivo growth and tumor suppressor pathway in the mammalian liver. Proc Natl Acad Sci USA. 2010; 107: 1437-42.

24. Lee KP, Lee JH, Kim TS, Kim TH, Park HD, Byun JS, et al. The Hippo-Salvador pathway restrains hepatic oval cell proliferation, liver size, and liver tumorigenesis. Proc Natl Acad Sci U S A. 2010; 107: 8248-53.

25. Maejima Y, Kyoi S, Zhai P, Liu T, Li H, Ivessa A, et al. Mst1 inhibits autophagy by promoting the interaction between Beclin1 and Bcl-2. Nat Med. 2013; 19: 1478-88.

26. Yuan F, Wang J, Li R, Zhao X, Zhang Y, Liu B, et al. A New Regulatory Mechanism Between P53 And YAP Crosstalk By SIRT1 Mediated Deacetylation To Regulate Cell Cycle And Apoptosis In A549 Cell Lines. Cancer Manag Res. 2019; 11: 8619-33.

27. Kurppa KJ, Liu Y, To C, Zhang T, Fan M, Vajdi A, et al. Treatment-Induced Tumor Dormancy through YAP-Mediated Transcriptional Reprogramming of the Apoptotic Pathway. Cancer Cell. 2020; 37: 104-22 e12.

28. Varelas X. The Hippo pathway effectors TAZ and YAP in development, homeostasis and disease. Development. 2014; 141: 1614-26.

29. Strano S, Monti O, Pediconi N, Baccarini A, Fontemaggi G, Lapi E, et al. The transcriptional coactivator Yes-associated protein drives p73 gene-target specificity in response to DNA Damage. Mol Cell. 2005; 18: 447-59.

30. Lehtinen MK, Yuan Z, Boag PR, Yang Y, Villen J, Becker EB, et al. A conserved MST-FOXO signaling pathway mediates oxidative-stress responses and extends life span. Cell. 2006; 125: 987-1001.

31. Yuan Z, Lehtinen MK, Merlo P, Villen J, Gygi S, Bonni A. Regulation of neuronal cell death by MST1-FOXO1 signaling. J Biol Chem. 2009; 284: 11285-92.

32. Du X, Shi H, Li J, Dong Y, Liang J, Ye J, et al. Mst1/Mst2 regulate development and function of regulatory $\mathrm{T}$ cells through modulation of Foxo1/Foxo3 stability in autoimmune disease. J Immunol. 2014; 192: 1525-35.

33. Meng F, Zhou R, Wu S, Zhang O, Jin O, Zhou Y, et al. Mst1 shuts off cytosolic antiviral defense through IRF3 phosphorylation. Genes Dev. 2016; 30: 1086-100.

34. Ma S, Meng Z, Chen R, Guan KL. The Hippo Pathway: Biology and Pathophysiology. Annu Rev Biochem. 2019; 88: 577-604

35. Meng Z, Moroishi T, Guan KL. Mechanisms of Hippo pathway regulation. Genes Dev. 2016; 30: 1-17.

36. Dupont S, Morsut L, Aragona M, Enzo E, Giulitti S, Cordenonsi M, et al. Role of YAP/TAZ in mechanotransduction. Nature. 2011; 474: 179-83.

37. Kim NG, Koh E, Chen X, Gumbiner BM. E-cadherin mediates contact inhibition of proliferation through Hippo signaling-pathway components. Proc Natl Acad Sci USA. 2011; 108: 11930-5.

38. Li Y, Zhou H, Li F, Chan SW, Lin Z, Wei Z, et al. Angiomotin binding-induced activation of Merlin/NF2 in the Hippo pathway. Cell Res. 2015; 25: 801-17.

39. Mo JS, Meng Z, Kim YC, Park HW, Hansen CG, Kim S, et al. Cellular energy stress induces AMPK-mediated regulation of YAP and the Hippo pathway. Nat Cell Biol. 2015; 17: 500-10.

40. Wang W, Xiao ZD, Li X, Aziz KE, Gan B, Johnson RL, et al. AMPK modulates Hippo pathway activity to regulate energy homeostasis. Nat Cell Biol. 2015; 17: 490-9.

41. Shao D, Zhai P, Del Re DP, Sciarretta S, Yabuta N, Nojima H, et al. A functional interaction between Hippo-YAP signalling and FoxO1 mediates the oxidative stress response. Nat Commun. 2014; 5: 3315.

42. Ganem NJ, Cornils H, Chiu SY, O'Rourke KP, Arnaud J, Yimlamai D, et al. Cytokinesis failure triggers hippo tumor suppressor pathway activation. Cell. 2014; 158: 833-48. 
43. Yu FX, Guan KL. The Hippo pathway: regulators and regulations. Genes Dev. 2013; 27: 355-71.

44. Peng Q, Li H, Chen J, Wang Y, Tang Z. Oral submucous fibrosis in Asian countries. J Oral Pathol Med. 2020; 49: 294-304.

45. Li J, Yao M, Zhu X, Li Q, He J, Chen L, et al. YAP-Induced EndothelialMesenchymal Transition in Oral Submucous Fibrosis. J Dent Res. 2019; 98 : 920-9.

46. Yao M, Li J, Yuan S, Zhu X, Hu Z, Li Q, et al. Role of the arecoline/YAP1/ BMP4 pathway in promoting endothelial-mesenchymal transition in oral submucous fibrosis. J Oral Pathol Med. 2020; 49: 305-10.

47. Sharma M, Hunter KD, Fonseca FP, Shetty SS, Radhakrishnan R. Role of Yes-associated protein and transcriptional coactivator with PDZ-binding motif in the malignant transformation of oral submucous fibrosis. Arch Oral Biol. 2021; 128: 105164.

48. Noguchi S, Saito A, Nagase T. YAP/TAZ Signaling as a Molecular Link between Fibrosis and Cancer. Int J Mol Sci. 2018; 19.

49. Zhou D, Zhang $\mathrm{Y}, \mathrm{Wu} \mathrm{H}$, Barry E, Yin $\mathrm{Y}$, Lawrence E, et al. Mst1 and Mst2 protein kinases restrain intestinal stem cell proliferation and colonic tumorigenesis by inhibition of Yes-associated protein (Yap) overabundance. Proc Natl Acad Sci U S A. 2011; 108: E1312-20.

50. Cheung P, Xiol J, Dill MT, Yuan WC, Panero R, Roper J, et al. Regenerative Reprogramming of the Intestinal Stem Cell State via Hippo Signaling Suppresses Metastatic Colorectal Cancer. Cell Stem Cell. 2020; 27: 590-604 e9.

51. Cordenonsi M, Zanconato F, Azzolin L, Forcato M, Rosato A, Frasson C, et al The Hippo transducer TAZ confers cancer stem cell-related traits on breast cancer cells. Cell. 2011; 147: 759-72.

52. Wang Y, Dong Q, Zhang Q, Li Z, Wang E, Qiu X. Overexpression of yes-associated protein contributes to progression and poor prognosis of non-small-cell lung cancer. Cancer Sci. 2010; 101: 1279-85.

53. Yoshida GJ. Regulation of heterogeneous cancer-associated fibroblasts: the molecular pathology of activated signaling pathways. J Exp Clin Cancer Res. 2020; 39: 112.

54. Omori H, Nishio M, Masuda M, Miyachi Y, Ueda F, Nakano T, et al. YAP1 is a potent driver of the onset and progression of oral squamous cell carcinoma. Sci Adv. 2020; 6: eaay3324.

55. Hiemer SE, Zhang L, Kartha VK, Packer TS, Almershed M, Noonan V, et al. A YAP/TAZ-Regulated Molecular Signature Is Associated with Oral Squamous Cell Carcinoma. Mol Cancer Res. 2015; 13: 957-68.

56. Peng Y, Hu S, Zhang K, Wang Y, Rouzi M, Zhou D, et al. Downregulation of MicroRNA-130a Inhibits Oral Squamous Cell Carcinoma Proliferation and Metastasis via the Hippo-YAP Pathway. Cancer Manag Res. 2021; 13: 4829-40.

57. García-Escudero $R$, Segrelles $C$, Dueñas $M$, Pombo $M$, Ballestín C, Alonso-Riaño $\mathrm{M}$, et al. Overexpression of PIK3CA in head and neck squamous cell carcinoma is associated with poor outcome and activation of the YAP pathway. Oral Oncol. 2018; 79: 55-63.

58. Sanchez-Vega F, Mina M, Armenia J, Chatila WK, Luna A, La KC, et al. Oncogenic Signaling Pathways in The Cancer Genome Atlas. Cell. 2018; 173: 321-37 e10.

59. Hasegawa K, Fujii S, Matsumoto S, Tajiri Y, Kikuchi A, Kiyoshima T. YAP signaling induces PIEZO1 to promote oral squamous cell carcinoma cell proliferation. J Pathol. 2021; 253: 80-93.

60. Li Z, Wang Y, Zhu Y, Yuan C, Wang D, Zhang W, et al. The Hippo transducer TAZ promotes epithelial to mesenchymal transition and cancer stem cell maintenance in oral cancer. Mol Oncol. 2015; 9: 1091-105.

61. Li J, Li Z, Wu Y, Wang Y, Wang D, Zhang W, et al. The Hippo effector TAZ promotes cancer stemness by transcriptional activation of SOX2 in head neck squamous cell carcinoma. Cell Death Dis. 2019; 10: 603

62. Sarode GS, Sarode SC, Sengupta N, Ghone U, Kumar Sharma N, Gadbail AR, et al. Denture induced mechanotransduction can contribute to oral carcinogenesis. Med Hypotheses. 2021; 148: 110507.

63. Bartucci M, Dattilo R, Moriconi C, Pagliuca A, Mottolese M, Federici G, et al. TAZ is required for metastatic activity and chemoresistance of breast cancer stem cells. Oncogene. 2015; 34: 681-90.

64. Baia GS, Caballero OL, Orr BA, Lal A, Ho JS, Cowdrey C, et al. Yes-associated protein 1 is activated and functions as an oncogene in meningiomas. Mol Cancer Res. 2012; 10: 904-13.

65. Cheng H, Zhang Z, Rodriguez-Barrueco R, Borczuk A, Liu H, Yu J, et al. Functional genomics screen identifies YAP1 as a key determinant to enhance treatment sensitivity in lung cancer cells. Oncotarget. 2016; 7: 28976-88.

66. Ciamporcero E, Shen H, Ramakrishnan S, Yu Ku S, Chintala S, Shen L, et al. YAP activation protects urothelial cell carcinoma from treatment-induced DNA damage. Oncogene. 2016; 35: 1541-53.

67. Lin L, Sabnis AJ, Chan E, Olivas V, Cade L, Pazarentzos E, et al. The Hippo effector YAP promotes resistance to RAF- and MEK-targeted cancer therapies. Nat Genet. 2015; 47: 250-6.

68. Yoshikawa $\mathrm{K}$, Noguchi $\mathrm{K}$, Nakano $\mathrm{Y}$, Yamamura $\mathrm{M}$, Takaoka $\mathrm{K}$ Hashimoto-Tamaoki T, et al. The Hippo pathway transcriptional co-activator, $\mathrm{YAP}$, confers resistance to cisplatin in human oral squamous cell carcinoma. Int J Oncol. 2015; 46: 2364-70.

69. Shriwas O, Arya R, Mohanty S, Mohapatra P, Kumar S, Rath R, et al. RRBP1 rewires cisplatin resistance in oral squamous cell carcinoma by regulating Hippo pathway. Br J Cancer. 2021; 124: 2004-16.

70. Chia S, Low JL, Zhang X, Kwang XL, Chong FT, Sharma A, et al. Phenotype-driven precision oncology as a guide for clinical decisions one patient at a time. Nat Commun. 2017; 8: 435.
71. Akervall J, Nandalur S, Zhang J, Qian CN, Goldstein N, Gyllerup P, et al. A novel panel of biomarkers predicts radioresistance in patients with squamous cell carcinoma of the head and neck. Eur J Cancer. 2014; 50: 570-81.

72. Eun YG, Lee D, Lee YC, Sohn BH, Kim EH, Yim SY, et al. Clinical significance of YAP1 activation in head and neck squamous cell carcinoma. Oncotarget. 2017; 8: 111130-43.

73. Luo J, Zhang L, Guo L, Yang S. PKM2 regulates proliferation and apoptosis through the Hippo pathway in oral tongue squamous cell carcinoma. Oncol Lett. 2021; 21: 461

74. Wei Z, Wang Y, Li Z, Yuan C, Zhang W, Wang D, et al. Overexpression of Hippo pathway effector TAZ in tongue squamous cell carcinoma: correlation with clinicopathological features and patients' prognosis. J Oral Pathol Med. 2013; 42: 747-54.

75. Luo HY, Li TJ. Odontogenic tumors: a study of 1309 cases in a Chinese population. Oral Oncol. 2009; 45: 706-11.

76. Shear $M$. The aggressive nature of the odontogenic keratocyst: is it a benign cystic neoplasm? Part 2. Proliferation and genetic studies. Oral Oncol. 2002; 38: 323-31.

77. Man QW, Ma YQ, Liu JY, Zhao Y, Liu B, Zhao YF. Expression of YAP/TAZ in Keratocystic Odontogenic Tumors and Its Possible Association with Proliferative Behavior. Biomed Res Int. 2017; 2017: 4624890.

78. Anand R, Sarode SC, Sarode GS, Rajpurohit LS, Patil S. Yes-associated protein (YAP) molecule expression in odontogenic epithelium of ameloblastoma. Clin Oral Investig. 2019; 23: 2389-94.

79. Gu Y, Han X. Toll-Like Receptor Signaling and Immune Regulatory Lymphocytes in Periodontal Disease. Int J Mol Sci. 2020; 21.

80. Wallet SM, Puri V, Gibson FC. Linkage of Infection to Adverse Systemic Complications: Periodontal Disease, Toll-Like Receptors, and Other Pattern Recognition Systems. Vaccines (Basel). 2018; 6 .

81. Lim KH, Staudt LM. Toll-like receptor signaling. Cold Spring Harb Perspect Biol. 2013; 5: a011247.

82. Newton K, Dixit VM. Signaling in innate immunity and inflammation. Cold Spring Harb Perspect Biol. 2012; 4

83. Deng Y, Lu J, Li W, Wu A, Zhang X, Tong W, et al. Reciprocal inhibition of YAP/TAZ and NF-kappaB regulates osteoarthritic cartilage degradation. Nat Commun. 2018; 9: 4564.

84. Lv Y, Kim K, Sheng Y, Cho J, Qian Z, Zhao YY, et al. YAP Controls Endothelial Activation and Vascular Inflammation Through TRAF6. Circ Res. 2018; 123: 43-56.

85. LaCanna R, Liccardo D, Zhang P, Tragesser L, Wang Y, Cao T, et al. Yap/Taz regulate alveolar regeneration and resolution of lung inflammation. J Clin Invest. 2019; 129: 2107-22

86. Kinane DF, Stathopoulou PG, Papapanou PN. Periodontal diseases. Nat Rev Dis Primers. 2017; 3: 17038

87. Kearns AE, Khosla S, Kostenuik PJ. Receptor activator of nuclear factor kappaB ligand and osteoprotegerin regulation of bone remodeling in health and disease. Endocr Rev. 2008; 29: 155-92.

88. Fili S, Karalaki M, Schaller B. Mechanism of bone metastasis: the role of osteoprotegerin and of the host-tissue microenvironment-related survival factors. Cancer Lett. 2009; 283: 10-9.

89. Yang B, Sun $\mathrm{H}, \mathrm{Xu} \mathrm{X}$, Zhong H, Wu Y, Wang J. YAP1 inhibits the induction of TNF-alpha-stimulated bone-resorbing mediators by suppressing the NF-kappaB signaling pathway in MC3T3-E1 cells. J Cell Physiol. 2020; 235: 4698-708.

90. Xiang $\mathrm{L}$, Zhang $\mathrm{X}, \mathrm{Yu} \mathrm{H}$, Wang $\mathrm{B}$, Lin $\mathrm{Z}$, Gong $\mathrm{P}$. Overexpression of alphaCGRP promotes osteogenesis of periodontal ligament cells by regulation of YAP signaling. J Cell Physiol. 2019; 234: 5077-85.

91. Pan W, Yang L, Li J, Xue L, Wei W, Ding H, et al. Traumatic occlusion aggravates bone loss during periodontitis and activates Hippo-YAP pathway. J Clin Periodontol. 2019; 46: 438-47.

92. Gurav AN. The implication of periodontitis in vascular endothelial dysfunction. Eur J Clin Invest. 2014; 44: 1000-9.

93. Alvarez C, Monasterio G, Cavalla F, Cordova LA, Hernandez M, Heymann D, et al. Osteoimmunology of Oral and Maxillofacial Diseases: Translational Applications Based on Biological Mechanisms. Front Immunol. 2019; 10: 1664

94. Diomede F, Marconi GD, Fonticoli L, Pizzicanella J, Merciaro I, Bramanti P, et al. Functional Relationship between Osteogenesis and Angiogenesis in Tissue Regeneration. Int J Mol Sci. 2020; 21.

95. Kim J, Kim YH, Kim J, Park DY, Bae H, Lee DH, et al. YAP/TAZ regulates sprouting angiogenesis and vascular barrier maturation. J Clin Invest. 2017; 127: 3441-61

96. Wang X, Freire Valls A, Schermann G, Shen Y, Moya IM, Castro L, et al. YAP/TAZ Orchestrate VEGF Signaling during Developmental Angiogenesis. Dev Cell. 2017; 42: 462-78 e7.

97. Azad T, Janse van Rensburg HJ, Lightbody ED, Neveu B, Champagne A Ghaffari A, et al. A LATS biosensor screen identifies VEGFR as a regulator of the Hippo pathway in angiogenesis. Nat Commun. 2018; 9: 1061.

98. Zhou A, Yu H, Liu J, Zheng J, Jia Y, Wu B, et al. Role of Hippo-YAP Signaling in Osseointegration by Regulating Osteogenesis, Angiogenesis, and Osteoimmunology. Front Cell Dev Biol. 2020; 8: 780.

99. Feng Y, Liang Y, Zhu X, Wang M, Gui Y, Lu Q, et al. The signaling protein Wnt5a promotes TGFbeta1-mediated macrophage polarization and kidney fibrosis by inducing the transcriptional regulators Yap/Taz. J Biol Chem. 2018; 293: $19290-302$ 
100. Li C, Jin Y, Wei S, Sun Y, Jiang L, Zhu Q, et al. Hippo Signaling Controls NLR Family Pyrin Domain Containing 3 Activation and Governs Immunoregulation of Mesenchymal Stem Cells in Mouse Liver Injury. Hepatology. 2019; 70: 1714-31.

101. Zhou X, Li W, Wang S, Zhang P, Wang Q, Xiao J, et al. YAP Aggravates Inflammatory Bowel Disease by Regulating M1/M2 Macrophage Polarization and Gut Microbial Homeostasis. Cell Rep. 2019; 27: 1176-89 e5.

102. Zhang Q, Wu B, Yuan Y, Zhang X, Guo Y, Gong P, et al. CGRP-modulated M2 macrophages regulate osteogenesis of MC3T3-E1 via Yap1. Arch Biochem Biophys. 2021; 697: 108697.

103. Zhu WQ, Ming PP, Qiu J, Shao SY, Yu YJ, Chen JX, et al. Effect of titanium ions on the Hippo/YAP signaling pathway in regulating biological behaviors of MC3T3-E1 osteoblasts. J Appl Toxicol. 2018; 38: 824-33.

104. Tang KM, Chen W, Tang ZH, Yu XY, Zhu WQ, Zhang SM, et al. Role of the Hippo-YAP/NF-kappaB signaling pathway crosstalk in regulating biological behaviors of macrophages under titanium ion exposure. J Appl Toxicol. 2021; 41: 561-71.

105. Walker JL, Menko AS, Khalil S, Rebustini I, Hoffman MP, Kreidberg JA, et al. Diverse roles of E-cadherin in the morphogenesis of the submandibular gland: insights into the formation of acinar and ductal structures. Dev Dyn. 2008; 237: 3128-41.

106. Enger TB, Samad-Zadeh A, Bouchie MP, Skarstein K, Galtung HK, Mera T, et al. The Hippo signaling pathway is required for salivary gland development and its dysregulation is associated with Sjogren's syndrome. Lab Invest. 2013; 93: 1203-18.

107. Szymaniak AD, Mi R, McCarthy SE, Gower AC, Reynolds TL, Mingueneau M, et al. The Hippo pathway effector YAP is an essential regulator of ductal progenitor patterning in the mouse submandibular gland. Elife. 2017; 6 .

108. Seo BM, Miura M, Gronthos S, Bartold PM, Batouli S, Brahim J, et al. Investigation of multipotent postnatal stem cells from human periodontal ligament. Lancet. 2004; 364: 149-55.

109. Wen Y, Ji Y, Zhang Y, Jiang B, Tang C, Wang Q, et al. Knockdown of Yes-Associated Protein Induces the Apoptosis While Inhibits the Proliferation of Human Periodontal Ligament Stem Cells through Crosstalk between Erk and Bcl-2 Signaling Pathways. Int J Med Sci. 2017; 14: 1231-40.

110. Jia L, Gu W, Zhang Y, Jiang B, Qiao X, Wen Y. Activated Yes-Associated Protein Accelerates Cell Cycle, Inhibits Apoptosis, and Delays Senescence in Human Periodontal Ligament Stem Cells. Int J Med Sci. 2018; 15: 1241-50.

111. Chen X, Wang Q, Gu K, Li A, Fu X, Wang Y, et al. Effect of YAP on an Immortalized Periodontal Ligament Stem Cell Line. Stem Cells Int. 2019; 2019: 6804036.

112. Gu K, Fu X, Tian H, Zhang Y, Li A, Wang Y, et al. TAZ promotes the proliferation and osteogenic differentiation of human periodontal ligament stem cells via the p-SMAD3. J Cell Biochem. 2020; 121: 1101-13.

113. Cargnello M, Roux PP. Activation and function of the MAPKs and their substrates, the MAPK-activated protein kinases. Microbiol Mol Biol Rev. 2011; 75: 50-83.

114. Hemmings BA, Restuccia DF. The PI3K-PKB/Akt pathway. Cold Spring Harb Perspect Biol. 2015; 7.

115. Jia L, Zhang Y, Ji Y, Xiong Y, Zhang W, Wen Y, et al. YAP balances the osteogenic and adipogenic differentiation of hPDLSCs in vitro partly through the Wnt/ $\beta$-catenin signaling pathway. Biochem Biophys Res Commun. 2019; 518: $154-60$.

116. Zheng L, Zhang L, Chen L, Jiang J, Zhou X, Wang M, et al. Static magnetic field regulates proliferation, migration, differentiation, and YAP/TAZ activation of human dental pulp stem cells. J Tissue Eng Regen Med. 2018; 12: 2029-40.

117. Du Y, Montoya C, Orrego S, Wei X, Ling J, Lelkes PI, et al. Topographic cues of a novel bilayered scaffold modulate dental pulp stem cells differentiation by regulating YAP signalling through cytoskeleton adjustments. Cell Prolif. 2019; 52: e12676.

118. Li Z, Ge X, Lu J, Bian M, Li N, Wu X, et al. MiR-141-3p regulates proliferation and senescence of stem cells from apical papilla by targeting YAP. Exp Cell Res. 2019; 383: 111562.

119. Zaidi SK, Sullivan AJ, Medina R, Ito Y, van Wijnen AJ, Stein JL, et al. Tyrosine phosphorylation controls Runx2-mediated subnuclear targeting of YAP to repress transcription. EMBO J. 2004; 23: 790-9.

120. Lin X, Yang H, Wang L, Li W, Diao S, Du J, et al. AP2a enhanced the osteogenic differentiation of mesenchymal stem cells by inhibiting the formation of YAP/RUNX2 complex and BARX1 transcription. Cell Prolif. 2019; 52: e12522.

121. Pan $\mathrm{H}, \mathrm{Xie} \mathrm{Y}$, Zhang $\mathrm{Z}, \mathrm{Li}, \mathrm{K}, \mathrm{Hu} \mathrm{D}$, Zheng $\mathrm{X}$, et al YAP-mediated mechanotransduction regulates osteogenic and adipogenic differentiation of BMSCs on hierarchical structure. Colloids Surf B Biointerfaces. 2017; 152: 344-53.

122. Wei Q, Holle A, Li J, Posa F, Biagioni F, Croci O, et al. BMP-2 Signaling and Mechanotransduction Synergize to Drive Osteogenic Differentiation via YAP/TAZ. Adv Sci (Weinh). 2020; 7: 1902931.

123. Wang B, Wu B, Jia Y, Jiang Y, Yuan Y, Man Y, et al. Neural peptide promotes the angiogenesis and osteogenesis around oral implants. Cell Signal. 2021; 79: 109873.

124. Yang Y, Wang BK, Chang ML, Wan ZQ, Han GL. Cyclic Stretch Enhances Osteogenic Differentiation of Human Periodontal Ligament Cells via YAP Activation. Biomed Res Int. 2018; 2018: 2174824.
125. Wu Y, Ou Y, Liao C, Liang S, Wang Y. High-throughput sequencing analysis of the expression profile of microRNAs and target genes in mechanical force-induced osteoblastic/cementoblastic differentiation of human periodontal ligament cells. Am J Transl Res. 2019; 11: 3398-411.

126. Sun B, Wen $Y$, Wu X, Zhang Y, Qiao X, Xu X. Expression pattern of YAP and TAZ during orthodontic tooth movement in rats. J Mol Histol. 2018; 49: 123-31.

127. Heallen T, Zhang M, Wang J, Bonilla-Claudio M, Klysik E, Johnson RL, et al. Hippo pathway inhibits Wnt signaling to restrain cardiomyocyte proliferation and heart size. Science. 2011; 332: 458-61.

128. Xin M, Kim Y, Sutherland LB, Qi X, McAnally J, Schwartz RJ, et al. Regulation of insulin-like growth factor signaling by Yap governs cardiomyocyte proliferation and embryonic heart size. Sci Signal. 2011; 4: ra70.

129. von Gise A, Lin Z, Schlegelmilch K, Honor LB, Pan GM, Buck JN, et al. YAP1, the nuclear target of Hippo signaling, stimulates heart growth through cardiomyocyte proliferation but not hypertrophy. Proc Natl Acad Sci USA. 2012; 109: 2394-9.

130. Kapoor A, Yao W, Ying H, Hua S, Liewen A, Wang Q, et al. Yap1 activation enables bypass of oncogenic Kras addiction in pancreatic cancer. Cell. 2014; 158: $185-97$.

131. Cottini F, Hideshima T, Xu C, Sattler M, Dori M, Agnelli L, et al. Rescue of Hippo coactivator YAP1 triggers DNA damage-induced apoptosis in hematological cancers. Nat Med. 2014; 20: 599-606.

132. Pavel M, Renna M, Park SJ, Menzies FM, Ricketts T, Fullgrabe J, et al. Contact inhibition controls cell survival and proliferation via YAP/TAZ-autophagy axis. Nat Commun. 2018; 9: 2961.

133. Zhou Y, Wang Y, Zhou W, Chen T, Wu Q, Chutturghoon VK, et al. YAP promotes multi-drug resistance and inhibits autophagy-related cell death in hepatocellular carcinoma via the RAC1-ROS-mTOR pathway. Cancer Cell Int. 2019; 19: 179 .

134. Liu Z, Zeng W, Wang S, Zhao X, Guo Y, Yu P, et al. A potential role for the Hippo pathway protein, YAP, in controlling proliferation, cell cycle progression, and autophagy in BCPAP and KI thyroid papillary carcinoma cells. Am J Transl Res. 2017; 9: 3212-23.

135. Aird KM, Zhang G, Li H, Tu Z, Bitler BG, Garipov A, et al. Suppression of nucleotide metabolism underlies the establishment and maintenance of oncogene-induced senescence. Cell Rep. 2013; 3: 1252-65.

136. Fu L, Hu Y, Song M, Liu Z, Zhang W, Yu FX, et al. Up-regulation of FOXD1 by YAP alleviates senescence and osteoarthritis. PLoS Biol. 2019; 17: e3000201.

137. Marti P, Stein C, Blumer T, Abraham Y, Dill MT, Pikiolek M, et al. YAP promotes proliferation, chemoresistance, and angiogenesis in human cholangiocarcinoma through TEAD transcription factors. Hepatology. 2015; 62: 1497-510.

138. Wei H, Wang F, Wang Y, Li T, Xiu P, Zhong J, et al. Verteporfin suppresses cell survival, angiogenesis and vasculogenic mimicry of pancreatic ductal adenocarcinoma via disrupting the YAP-TEAD complex. Cancer Sci. 2017; 108: 478-87.

139. Song R, Gu D, Zhang L, Zhang X, Yu B, Liu B, et al. Functional significance of Hippo/YAP signaling for drug resistance in colorectal cancer. Mol Carcinog. 2018; 57: 1608-15.

140. Song S, Honjo S, Jin J, Chang SS, Scott AW, Chen Q, et al. The Hippo Coactivator YAP1 Mediates EGFR Overexpression and Confers Chemoresistance in Esophageal Cancer. Clin Cancer Res. 2015; 21: 2580-90.

141. Jamal B, Sengupta PK, Gao ZN, Nita-Lazar M, Amin B, Jalisi S, et al. Aberrant amplification of the crosstalk between canonical Wnt signaling and $\mathrm{N}$-glycosylation gene DPAGT1 promotes oral cancer. Oral Oncol. 2012; 48: 523-9.

142. Varelas X, Miller BW, Sopko R, Song S, Gregorieff A, Fellouse FA, et al. The Hippo pathway regulates Wnt/beta-catenin signaling. Dev Cell. 2010; 18: 579-91. 